\title{
Metamorfosis Bancaria en tiempos de COVID-19: Reflexiones para la Gerencia del
} Siglo XXI

\section{Banking Metamorphosis in times of COVID-19: Reflections for the Management of the 21 St Century}

\author{
Raysa Chirino-García \\ rchirino@urbe.edu.ve \\ Universidad Privada Dr. Rafael Belloso Chacín, Zulia \\ Venezuela \\ https://orcid.org/0000-0002-0336-2870 \\ Melquiades Salas-Anteliz \\ melquiadessalas@mail.uniatlantico.edu.co \\ Universidad del Atlántico, Atlántico \\ Colombia \\ https://orcid.org/0000-0002-3161-2400 \\ Wilfred Vilardy-Naggles \\ wilfred.vilardy@uac.edu.co \\ Universidad autónoma del Caribe, Barranquilla \\ Colombia \\ https://orcid.org/0000-0001-5201-6539
}

Recibido: 30 de mayo de 2020

Aprobado: 15 de agosto de 2020 


\section{RESUMEN}

El artículo de revisión tuvo el propósito de analizar la transformación bancaria en tiempos de COVID-19 presumiendo un reto empresarial para la gerencia del siglo XXI, afectada por un enemigo invisible, donde la oferta de servicios financieros esté en concordancia con los Decretos Gubernamentales que establecían el distanciamiento social y aislamiento. El estudio se fundamentó principalmente en la indagación introspectiva reflexiva, desde una perspectiva analítica y de naturaleza documental. En este sentido, se hizo un análisis reflexivo sobre las herramientas digitales implementadas para la automatización de transacciones financieras apoyadas en plataformas web, aplicaciones móviles, así como otras herramientas digitales que proporcionen ciberseguridad a los usuarios y a la banca. Concluyéndose que, el sector bancario ha experimentado una metamorfosis o transmutación frente al COVID-19; por lo que, la gerencia del siglo XXI debe lograr responsablemente las garantías necesarias, para una relación eficiente mediante comunicación efectiva y eficaz en el binomio banca-clientes.

Descriptores: Digitalización; administración financiera; gobierno electrónico. (Palabras del Tesauro UNESCO).

\section{ABSTRACT}

The review article had the purpose of analyzing the banking transformation in times of COVID-19, presuming a business challenge for the management of the XXI century, affected by an invisible enemy, where the supply of financial services is in accordance with the Government Decrees that established social distancing and isolation. The study was based mainly on reflective introspective inquiry, from an analytical and documentary perspective. In this sense, a reflective analysis was made of the digital tools implemented for the automation of financial transactions supported by web platforms, mobile applications, as well as other digital tools that provide cybersecurity to users and banks. Concluding that, the banking sector has undergone a metamorphosis or transmutation in the face of COVID-19; Therefore, the management of the 21 st century must responsibly achieve the necessary guarantees for an efficient relationship through effective and efficient communication in the banking-client binomial.

Descriptors: Digitization; financial administration; electronic governance. (Words from UNESCO Thesaurus). 


\section{INTRODUCCIÓN}

La llegada del Siglo XXI ha traído significativos adelantos tecnológicos, descubrimientos e innovaciones que a nivel industrial hacen perentoria la inclusión de nuevas tecnologías. Esto, representa una ruptura de paradigmas; además de constituirse en el punto de partida para la interconexión como soporte de los procesos de automatización, así como la digitalización de los mismos, desarrollando una trilogía entre las tecnologías de la información, informática y los softwares en las organizaciones.

Desde esta perspectiva, aunada a la situación de limitaciones globales impuestas por un enemigo invisible que ha causado una pandemia sin precedentes, resulta obligatorio la realización del trabajo en red conocido también como "home office" donde el uso de redes de información y comunicación coadyuven al distanciamiento social establecido por los organismos internacionales en materia de salud pública. Esto, indubitablemente, origina una modernización que da paso a una nueva filosofía de consumo, transacciones y actividades en general, requiriendo otras modalidades de producción para satisfacer mercados, así como diferentes maneras de hacer llegar lo que se fabrica y que facilite el acceso a bienes, productos y servicios; dado que, ante el escenario de contracción económica, los mercados deben ajustarse a las demandas de los usuarios para satisfacer las necesidades.

Es por ello, que Bueno Campos, Longo Somoza, Paz Salmador, \& Morcillo (2017), advierten que la Gerencia del siglo XXI se enfrenta al reto tecnológico, donde la banca transita por la necesidad de establecer un nuevo enfoque de negocio que propenda a una cadena de valor innovadora, fundamentada en la perspectiva de economía colaborativa con el fin de poder adaptarse a los cambios del entorno, generando nuevas ventajas competitivas basadas en la tecnología e innovación.

En otro orden de ideas, Massanell (2016) comenta que, el proceso de transformación de la banca pasa por el reforzamiento de la posición del cliente como único referente de la cultura corporativa; puesto que, es el cliente quien juzga, compara y hace las exigencias 


\section{Raysa Chirino-García; Melquiades Salas-Anteliz; Wilfred Vilardy-Naggles}

para que sus necesidades sean satisfechas. De ahí que, se hace relevante el urgente rediseño de la oferta de productos y servicios tendientes a un modelo coherente con la percepción de valor de los clientes, ya que resulta relevante lograr que expresen sus ideas y criticas para mejorar el servicio, utilizando para ello los canales digitales.

En cuanto a los cambios que están experimentando las instituciones financieras y de manera particular la banca, propenden a que las circunstancias actuales de pandemia por Covid-19, ponen a prueba los servicios ofrecidos, así como la metodología de trabajo, dado que el mercado demanda rapidez, eficacia y eficiencia. En este escenario, el uso de nuevas tecnologías por parte de la banca ha de contribuir de manera significativa a mitigar el contagio del COVID-19; puesto que, el distanciamiento social representa uno de los elementos esenciales para lograr la desaceleración de la propagación de virus y poder reactivar la economía así como las actividades empresariales, fundamentales para el desarrollo y pervivencia del país.

Aquí, es importante traer a colación a Avendaño (2018) cuando comenta que a través de servicios de banca electrónica, el sector financiero asume retos a razón de que requiere establece directrices para determinar las operaciones y servicios digitales necesarios, además de los medios que le permitan la identificación de las responsabilidades, las necesidades del usuario y la creación, transmisión, modificación o extinción de derechos así como obligaciones, inherentes a las operaciones y servicios que se traten.

Ahora bien, el sector empresarial ha tendido que efectuar cambios en las tecnologías, lo que apareja importantes decisiones estratégicas; puesto que, asume un rol relevante en la forma de gestionar los negocios, evidenciándose una transformación en la economía tradicional para dar paso a lo que hoy llamamos economía digital (Fanjul \& Valdunciel, 2007). De ahí que, los modelos de estrategia empresarial convencionales han transitado hacia nuevos paradigmas, los cuales han reconfigurado las estrategias empresariales, aunque ha representado un desafío para la gerencia puesto que se observa una segmentación; de manera que el impacto de esta "nueva economía" por sectores está 


\section{Raysa Chirino-García; Melquiades Salas-Anteliz; Wilfred Vilardy-Naggles}

siendo desigual. No obstante, el sector financiero ha sido pionero en el uso de internet como nuevo canal de distribución de productos financieros, el cual no exige un intercambio físico de bienes, ya que el dinero se convierte en un bien electrónico. En otras palabras, el uso de canales digitales en la banca permite conseguir un crecimiento sostenido.

Desde otra perspectiva, Pejić-Bach (2021), señala que en estos momentos de crisis ocasionada por el Covid-19, se han impuesto cuarentenas masivas a nivel mundial que detuvieron la mayoría de las interacciones entre las personas, lo que popularmente se conoce como distanciamiento social, ello implica la reducción del contacto físico entre las personas. Sin embargo, ello no ha impedido que los seres humanos permanezcan virtualmente conectados, gracias a la abundancia de diversas soluciones digitales ofrecidas en el mercado.

En este contexto, las diversas teorías de aceptación de la tecnología deberían modificarse o rediseñarse, teniendo en cuenta que en estos tiempos de crisis, los usuarios tienen una percepción diferente de la tecnología; en otras palabras, el abordaje de sus contribuciones prácticas debe considerar varios aspectos sobre el uso a corto, mediano y largo plazo de las soluciones electrónicas en tiempos de Covid-19, donde la comunicación juega un rol significativo para la gerencia (Pejić-Bach, 2021). En relación con este último, la gerencia del siglo XXI se enfrenta al reto proveniente de la transformación bancaria, dado que, los usuarios se enfrentan a desorientación y ansiedad producto de cuestionarse ¿Qué tan seguros son los sistemas bancarios? ¿Qué tan educados y concientizados está la clientela o usuarios? En esta instancia, el sector deberá trabajar para incrementar los niveles de educación y consciencia, indispensables en el proceso de transformación.

A lo anterior se añade que, la mayoría de las operaciones deben realizarse de manera digital, puesto que, los trámites en estos momentos no pueden efectuarse de manera presencial; evidenciándose con ello que de la crisis emerge la oportunidad, ya que el 


\section{Raysa Chirino-García; Melquiades Salas-Anteliz; Wilfred Vilardy-Naggles}

usuario tiene respuesta en tiempo real de todo aquello que acontece en sus finanzas sin tener que movilizarse de su casa.

De ahí que, resulta necesario que el reajuste que efectúe la gerencia no solo sea a los canales digitales, sino también, respecto a la implementación de herramientas, aplicaciones, operaciones de inversión, instrumentos financieros, entre otros, para que las transacciones digitales se conviertan en las preferidas de los clientes, lo cual apoyaría las estrategias establecidas en materia sanitaria por la Organización Mundial de la Salud (OMS).

Con base a lo anterior, la presente investigación busca dar respuesta a la interrogante ¿Cómo es la metamorfosis Bancaria en tiempos de COVID-19 que permitan a la gerencia del siglo XXI enfrentar la pandemia? Puesto que, los bancos como instituciones financieras han debido implementar con premura procesos inteligentes que propicien actividades a menor costo, con celeridad, flexibles, eficientes y seguras, lo cual evidencia la transformación o metamorfosis de la banca tradicional (física) a una banca digital, constituyéndose en estos tiempos un reto para la gerencia del siglo $\mathrm{XXI}$, considerando que se deberán diseñar nuevos canales de información y comunicación, para enfrentar los cambios forzosos producto de la pandemia, al mismo tiempo que existen limitaciones vinculados a una conectividad deficiente, además de un deteriorado servicio de electricidad en conjunto con otros factores. Volviendo la mirada a lo anterior, el presente estudio procura analizar la transformación bancaria en tiempos de COVID-19 presumiendo un reto organizacional para la gerencia del siglo XXI.

\section{MÉTODO}

La investigación se fundamentó principalmente en la indagación introspectiva reflexiva, desde una perspectiva analítica a propósito de construir nuevos conocimientos (Aldana Zavala, 2019). Asimismo, se caracteriza por ser un estudio de naturaleza documental, pues de lo que se trata, es de indagar en el tema de la transformación bancaria en tiempos de COVID-19 presumiendo un reto organizacional para la gerencia del siglo XXI; a través 
de un proceso sistemático de búsqueda, recogida, organización, análisis, crítica e interpretación de datos e informaciones obtenidos de fuentes documentales: impresas, audiovisuales y/o electrónicas, en relación a un tema concreto.

Al respecto, Arias (2012) señala que la investigación documental se basa en la producción y análisis de datos procedentes de materiales impresos y de cualquier tipo de documento. Del mismo modo, para Fontaines (2012, p.130) comenta que la modalidad documental se orienta hacia "la recolección de datos mediante el manejo de documentos de diversa índole". Por consiguiente, se procedió a la búsqueda de información preliminar en fuentes bibliográficas, escritas y electrónicas referidas al tema de estudio, para organizar y desarrollar el presente análisis.

Por su parte, Palella y Martins (2015) señalan que en este tipo de investigación se utilizan documentos, que se recolectan, seleccionan, analizan y se presentan resultados de manera coherente; desplegándose, por parte del investigador, procedimientos lógicos y mentales como: el análisis, la síntesis, la deducción, la inducción, entre otros; y aplicándose diferentes técnicas de localización y fijación de datos, análisis de documentos y de contenidos. En tal sentido, y para procesar la información recolectada y seleccionada, fue necesario efectuar el análisis de contenido teórico mediante el manejo de mapas cognitivos (mentales y conceptuales) y el análisis semántico; pues son vías que llevan a la comprensión de los textos y documentos examinados.

\section{ANÁLISIS}

\section{Desafíos gerenciales en tiempos de COVID-19}

La crisis de COVID-19 es un evento pandémico mundial sin precedentes, que ha ocasionado un impacto calamitoso en la vida cotidiana, así como en la economía en general. En los mercados globales, la pandemia ha provocado una disrupción importante con inestabilidad, además, de críticas fluctuaciones en los volúmenes del comercio, lo 


\section{Raysa Chirino-García; Melquiades Salas-Anteliz; Wilfred Vilardy-Naggles}

cual ocasiona desafíos en diferentes sectores de la economía tales como: primarios, secundarios, de financiación y de derivados.

En este contexto, Boyle \& Nichols (2020) comentan que, el avance del COVID-19 y sus efectos, la crisis global, progresa. En consecuencia, la pandemia ha dejado al descubierto vulnerabilidades y oportunidades que tienen las organizaciones, sobre todo las del ámbito financiero y bancario, las cuales deben ser abordadas inevitablemente; puesto que, es preciso mayor resiliencia, eficiencia y eficacia para este sector. De manera que, de las circunstancias vividas se pueden extraer aprendizajes que sirvan como paliativo generalizado en la economía global; caso concreto, el sector bancario y financiero, el cual prevé el funcionamiento de un entorno laboral desde la casa o "home office" puesto que el desplazamiento laboral a corto plazo no será posible, ocasionando problemas relevantes en el ámbito operativos, culturales y comerciales.

Desde esta óptica, se puede decir que el COVID-19 está acelerando la digitalización de procesos administrativos y gerenciales de las organizaciones a nivel mundial, lo que representa un reto para el gobierno corporativo. Por consiguiente, estas deberán convenir sus objetivos y reajustarlos, teniendo en cuenta las lecciones aprendidas hasta ahora en esta pandemia y con miras a modificar ampliamente sus programas estratégicos, promocionando no solo la inclusión financiera sino la producción; de manera que, la gerencia de las organizaciones, forzosamente, deberá invertir para avanzar a un modelo más ágil, dando paso al Fintech con funciones auxiliares que coadyuven a mejorar y satisfacer al cliente.

En concreto, la Gerencia deberá incluir como factores claves para estimar una posible normalidad, la habilitación digital de los proceso y de la fuerza laboral, la identificación de la vulnerabilidad del negocio desde la óptica del rendimiento, resiliencia, así como el control de los resultados de la pandemia, el diseño de estrategias para el retorno y/o reactivación laboral que incluya prioridades estratégicas, además de determinar las oportunidades y amenazas que ello apareja en una "nueva normalidad". 
Raysa Chirino-García; Melquiades Salas-Anteliz; Wilfred Vilardy-Naggles

\section{Perspectiva mundial de la Banca Digital}

En la actualidad, el audaz y vanguardista entrelazamiento de técnicas de producción con sistemas inteligentes que se están incorporando vertiginosamente a las organizaciones y personas, están haciendo que emerja la cuarta Revolución Industrial; de manera que, la innovación fundamentada en la inteligencia artificial, la robotización, la interconexión digital, la industria conectada, donde las barreras entre el mundo físico y el digital son imprecisas, dan paso a un nuevo modelo económico que tiene efectos en todas las actividades del mercado financiero, (Antúnez, 2019).

En este escenario, el sector bancario a nivel mundial, ha implementado algunas estrategias de transformación digital, dado que la mayoría de ellos comenzaron a evidenciar los beneficios por parte de sus clientes en cuanto al uso de canales de autoservicio más económicos. Tal idea es confirmada en el informe mundial Capgemini (2016) sobre la Banca de Consumo, pues, se muestra la rapidez con la que se han modificado las preferencias de los clientes, siendo la banca a través de dispositivos móviles la que se lleva el 33\% de las interacciones de los clientes frente al 22\% en 2014. Asimismo, la usabilidad de estos canales, toda vez que analiza y expone que más del $70 \%$ del segmento más joven de Estados Unidos (USA) ha utilizado servicios de banca móvil en los últimos 12 meses, en comparación con solo el $40 \%$ del resto de la población adulta; considerando que alrededor del $94 \%$ de los pertenecientes a la generación del milenio son usuarios activos de la banca en línea (Nava, Karp \& Nash-Stacey, 2014). Cabe decir, que actualmente la proporción de uso de las instituciones financieras por parte de los jóvenes y los clientes más adultos se presenta mucho más pareja; puesto que, el mismo sistema ha conllevado a la adaptación de nuevos productos y procesos; por cuanto, cada día se da una mayor apropiación del entorno digital, obligando a las personas a readaptarse y formar parte de ella, obviando la resistencia al cambio de la plataforma bancaria, so pretexto de una presunta complejidad en el sistema. 
Raysa Chirino-García; Melquiades Salas-Anteliz; Wilfred Vilardy-Naggles

El alto componente tecnológico que ha irrumpido en el sector financiero, ha generado nuevas demandas de los servicios bancarios, exigiendo cambios profundos. Para ello, la banca se encuentra respondiendo al reto de la digitalización con diversas aproximaciones, a distintas velocidades; puesto que, no todas las empresas comprenden el significado de metamorfosis bancaria para convertirse en un banco digital. No obstante, los hábitos cambiantes de los consumidores, así como el nuevo entorno competitivo constriñen a la banca a afrontar con premura su digitalización para no quedarse atrás en un mercado financiero cambiante. Cabe decir, que en este proceso de transformación digital de la banca, se identifican tres etapas consecutivas: La primera, de desarrollo de nuevos canales y productos; la segunda, de adaptación de la infraestructura tecnológica; y la última, de cambios profundos en la organización para posicionarse estratégicamente en el entorno digital (Cuesta, Ruesta, Tuesta \& Urbiola, 2015).

No obstante, también se observa, que en la banca digital, los medios de pago y la irrupción de nuevas plataformas, es un proceso sujeto a múltiples mitos a veces alejados de la realidad más contrastable. En este sentido, la digitalización financiera transita por la adopción similar experimentada en otros países (Dinamarca, Islandia y Suecia), donde los instrumentos de pago han jugado un papel destacado, generándose un impacto potencial destacable no solo como alternativa a los servicios bancarios; sino, también, como parte y/o complemento de estos. Por lo tanto, hay que tener en cuenta que, en las transformaciones, los servicios basados en la digitalización no solo pueden acelerar cambios de orden económico, sino que también trae otros beneficios y reajustes sociales (Carbó, 2017).

Finalmente, se puede advertir que la digitalización podría apoyar a superar los costos, así como barreras físicas que obstaculizan una mayor inclusión financiera de segmentos de la población menos favorecida, desde diferentes perspectivas. Además, de producir cambios en la esfera privada fundamentalmente; pero, en los que la regulación tiene 
mucho que decir para conservar la perdurabilidad financiera, la protección del consumidor y asegurar un entorno competitivo equitativo entre los diferentes proveedores.

\section{De la Banca física o tradicional a la Banca Digital}

Para comprender hacia dónde va la banca hoy días, es importante dar un vistazo a su evolución en el tiempo a razón de la introducción de nuevas tecnologías. En este sentido, el desarrollo y avance de la banca se encuentra dividida en etapas, las cuales se enuncian a continuación:

- I etapa. Desarrollada en los años sesenta, cuyos objetivos eran la reducción de los costos, mejorar la productividad y brindar mayor seguridad. Con restricciones derivadas de las propias limitaciones informáticas de la época y de la falta de capacitación del personal que trabajaba en las entidades para ese momento.

- Il etapa. Se da durante los años setenta y con la introducción del teleproceso en el negocio bancario, cuyo propósito era mejorar el servicio ofrecido a los clientes y conseguir una mayor rapidez en la gestión del negocio bancario, sus limitaciones estaban referidas a las fallas del sistema de telecomunicaciones y a la homologación de normativas.

- III etapa. Vivida en los años ochenta, se obtuvo una mayor autonomía, nuevos puntos de venta, introducir la ofimática (aplicación de la informática al trabajo de oficina) y el sistema de banca electrónica. Las limitaciones presentadas fueron aquellos derivadas de los servicios telemáticos, de las incompatibilidades de los equipos y de los vacíos legales.

- IV etapa. En los inicios de los años noventa, se introduce el sistema de banca online o virtual, a través del cual se persigue la descentralización de muchas funciones, la reducción de puntos de venta, así como la comunicación y distribución a través de la red. Los problemas detectados, se relacionaron con lagunas legales en cuanto a seguridad en las operaciones. 
Universidad Nacional Experimental Francisco de Miranda (UNEFM). Santa Ana de Coro. Venezuela

\section{Raysa Chirino-García; Melquiades Salas-Anteliz; Wilfred Vilardy-Naggles}

- V etapa. Se desarrolla actualmente en las instituciones financieras, en donde el cliente tiene acceso a sus recursos a través de sucursales, internet y los teléfonos inteligentes, cuyas grandes bondades son: el ahorro del tiempo y la ejecución de operaciones interconectadas; lo que permite realizar todo tipos de gestiones bancarias desde cualquier lugar del mundo y a cualquier hora del día (Avendaño, 2018).

En este contexto de la migración bancaria, Reboiro (2020) señala que la banca tradicional ha tenido que redefinirse estratégicamente para acercarse a las nuevas entidades supeditadas a lo digital que han emergido; pues este proceso gradual de digitalización del sector bancario y financiero ha forjado la creación de organizaciones bancarias nativas digitales; es decir, con estructuras organizativas adecuadas mas al sector tecnológico que al financiero.

A propósito de lo planteado y trayendo a colación los planteamientos de Hempel \& Tur (2016), se puede decir que la digitalización de los procesos bancarios abarca los servicios prestados por la institución; asimismo, y en el marco de garantizar la eficiencia y seguridad de los sistemas de pago, se debe asegurar una operativa sencilla y sin sobresaltos de dichos sistemas. Sin embargo, se debe considerar que la introducción de innovaciones en los pagos y servicios asociados a las operaciones financieras, no previene nuevas fuentes de fragmentación en el mercado. Pues, la finalidad de la utilización de medios electrónicos en los sistemas de pago, propende a dinamizar los procesos basados en la digitalización con estándares comunes que coadyuven la interoperatividad.

Desde esta perspectiva, la digitalización impulsa el sector de los pagos al poder producir importantes cambios en este ecosistema, donde los nuevos actores van a adueñarse de una parte relevante de la cadena de valor. No obstante, esto puede originar riesgos en la desintermediación y pérdida de la relación con el cliente por parte de los bancos y 
Raysa Chirino-García; Melquiades Salas-Anteliz; Wilfred Vilardy-Naggles

operadores tradicionales, representando esto una posible amenaza, por la cual, los bancos deben reaccionar revisando y poniendo la máxima atención en las estrategias y alianzas actuales empleada para los pagos (Tramazaygues, 2016).

En complemento a lo abordado, (Gutiérrez, 2016), señala que los cambios en los hábitos de la clientela y la emergencia de nuevos competidores, demandan renovar de forma drástica la productividad del sector bancario. En este sentido, los avances de las llamadas tecnologías exponenciales y su ritmo de adopción, así como la regulación, se destacan como los principales factores que van a determinar la nueva estructura, y la velocidad con la que se configure, el sistema financiero. En resumen, estos representan los rasgos fundamentales de la transformación que los bancos actuales deben abordar para aspirar a una posición relevante en el nuevo sistema financiero.

Es conveniente resaltar que, el progreso digital no solo aporta mayor comodidad en los servicios bancario al proporcionar una mayor comunicación con el cliente; pues, también perpetra, consecuencialmente, la transformación de la cultura bancaria, reconociendo la coexistencia de dos mundos bancarios totalmente paralelos con el mismo cliente. En este contexto, Avendaño (2018), expresa que la evolución en el uso de las tecnologías implica la desaparición de límites geográficos y conceptuales de la industria bancaria; por cuanto, esto consigue trasladar la naturaleza misma desde lo tradicionalmente considerado, hasta la industria de distribución de la información relacionada con los servicios del sector financiero.

En otro tenor, Barrutia \& Echebarria, (2001), comentan que las entidades bancarias requieren desarrollar estrategias que coadyuve una ventaja del nuevo entorno; de ahí que, el Internet representa una nueva vía de transformación; y, previsiblemente, sea la vertiente más relevante en los actuales momentos. Igualmente, las instituciones bancarias son usuarios de una nueva tecnología que aporta valor, aunado a que estas pueden aprovechar el alto ritmo de expansión del comercio electrónico, participando directamente en él, en las vertientes de empresa- consumidor, entre empresas y como 
inversor en iniciativas relacionadas con la nueva economía, tal como se ilustra en el cuadro No. 2, en relación a lo que pueden hacer las entidades bancarias como usuarios de las nuevas tecnologías.

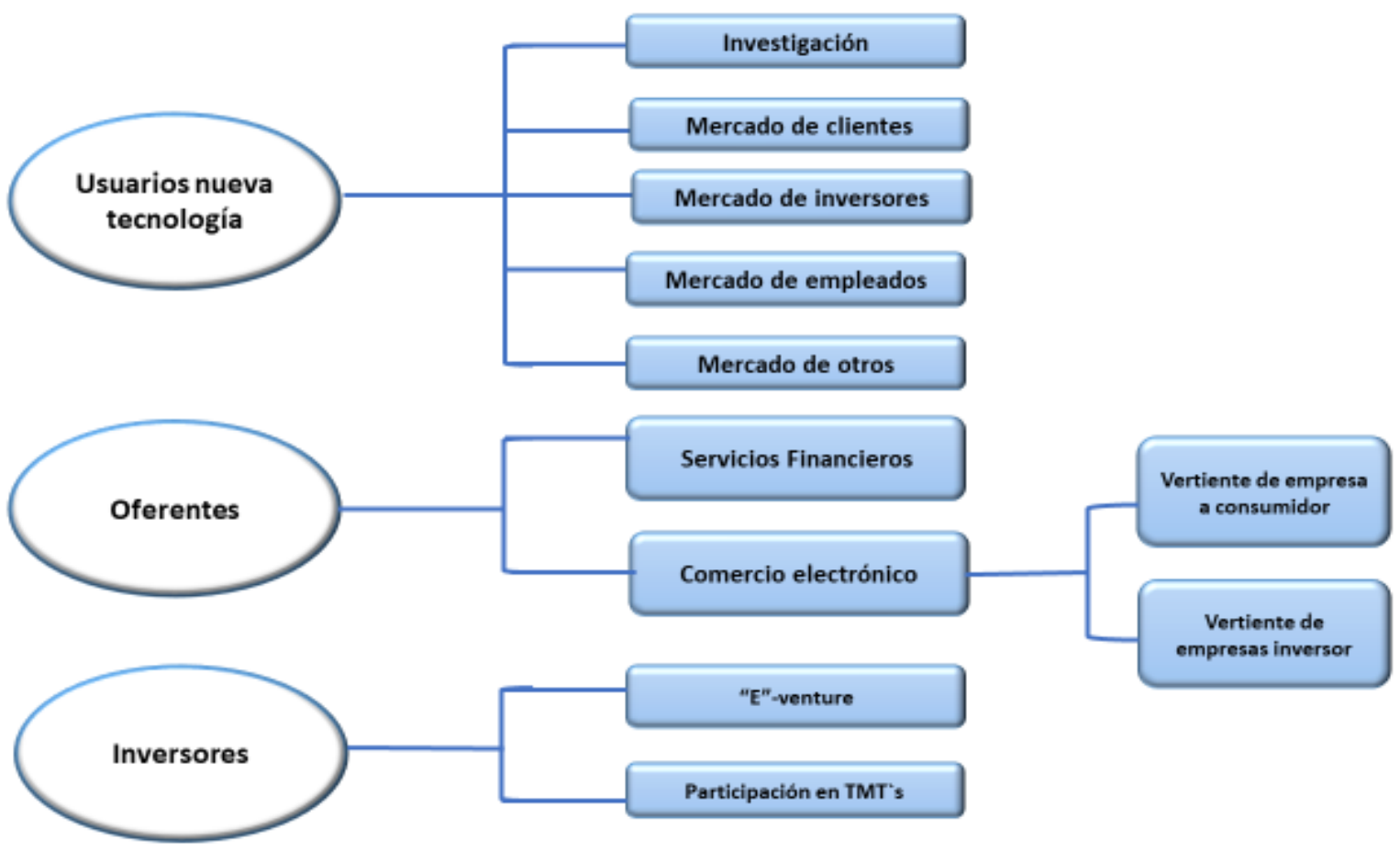

Figura.1. Posibilidades que ofrece la nueva economía a la banca tradicional. Fuente: Adaptación de Barrutia \& Echebarria (2001).

\section{Las Fintech y Bigtech}

Manifiesta Igual-Molina (2018), que el sector bancario experimenta una intensa transformación en su actividad, tanto es así, que ha tendido que reinventarse para atender las exigencias de los usuarios en cuanto a productos digitales, además de ajustar, en la mayoría de los casos, sus estructuras ineficientes. De esta manera, el sector financiero ha sido un ámbito casi exclusivo para las entidades financieras, pero la 
Raysa Chirino-García; Melquiades Salas-Anteliz; Wilfred Vilardy-Naggles

desvalorización de las tecnologías utilizadas ha permitido el surgimiento de nuevos interfaces en la industria, como las Fintech y Bigtech.

Resulta relevante efectuar la distinción entre Fintech y Bigtech. Al respecto, Vives (2019) señala, que el término Fintech es la suma etimológica de las palabras inglesas finance y technology, generalmente es utilizado para denominar a las empresas de tecnologías financieras; es decir, que ofrecen productos y servicios financieros mediante las tecnologías de la información y comunicación, como páginas de internet, redes sociales, así como aplicaciones para celulares, por otra parte, la banca tradicional no admite bien el término.

Aquí, es oportuno resaltar que las plataformas Fintech han logrado atender las exigencias no satisfechas de los clientes en materia de pagos, así como transferencias, crédito y asesoramiento de inversión. Tal es el caso de las generaciones jóvenes, que son más proclives al uso de los productos y servicios Fintech de los bancos digitales; pues juegan un rol relevante los factores demográficos y el daño notorio sufrido por los bancos tradicionales, (Vives, 2019). A lo planteado, se añade que las Fintech podrían beneficiarse de la marca establecida, las economías de escala y los canales de distribución de los operadores tradicionales. Sin embargo, el escenario de entrada de nuevos bancos (con licencia) es menos probable, habida cuenta de los elevados costes de cumplimiento legales asociados (Vives, 2019).

Cabe resalta que, el sector bancario ha transitado por una intensa transformación en sus actividades; tanto es así, que debe reinventarse para que con el cambio pueda atender las exigencias de los usuarios en cuanto a productos digitales, además de ajustar, en la mayoría de los casos, sus estructuras un tanto obsoletas e ineficientes. De esta manera, los productos y servicios ofrecidos por el sector financiero ha sido casi exclusivo para las entidades financieras, pero la desvalorización producto de lo acelerado de los cambios tecnológicos ha permitido el surgimiento de nuevos interfaces en la industria. 
En este sentido, las Fintech representan una alternativa en todos los ámbitos de la actividad financiera, mediante la modernización de fórmulas, tales como: mobile-first y data-driven, entre otras. Para dar amplitud a las cualidades ofrecidas por la plataforma Fintech, en el próximo cuadro se ilustran las ventajas y desventajas:

\section{Tabla 1.}

Ventajas y desventajas de las Fintech.

\section{BENEFICIOS}

Tecnología superior sin sistemas heredados; operativa simplificada.

Interfaz intuitiva y nuevo estándar de experiencia para el consumidor.

\section{Enfoque en segmentos de} negocio/actividades con mayor ROE.

Más financiación mediante recursos propios.

Capaces de atraer al mejor talento.

\section{INCONVENIENTES}

Ausencia de una base de clientes instalada y fiel.

Acceso limitado a información blanda

Falta de reputación y reconocimiento de marca.

Elevado coste del capital y reducido tamaño de su balance.

Falta de experiencia y pericia en gestión del riesgo y navegación de la regulación.

Sin acceso al prestamista de última instancia, sin licencia bancaria.

Fuente: Adaptación de Vives (2019) 
Raysa Chirino-García; Melquiades Salas-Anteliz; Wilfred Vilardy-Naggles

Pacheco \& Urbiola (2020), señalan que la transformación digital de los servicios financieros aunada a la incorporación de las Fintech dentro de los ecosistemas digitales, prepara la apertura competitiva de nuevos mercados que coadyuven a controlar los riesgos, promover la competencia e innovación. En este sentido, las Bigtech se están posicionando y pueden cambiar de forma significativa la estructura de la industria financiera; por lo tanto, estas plataformas representan una oportunidad de innovación para la banca, de igual medida que su magnitud y naturaleza de los riesgos.

Con respecto a las Bigtech, estas son una extensión de las Fintech; sin embargo, se diferencian ya que son grandes empresas tecnológicas con una red de operaciones bien establecidas que están ofreciendo servicios financieros por estar posicionadas y ser decisivas en el mundo de la economía digital. Cabe resaltar que Casilda (2020), comenta que las Bigtech, podrían controlar en el futuro cercano casi 9 billones de euros; tal es el caso, que hoy día los bancos comerciales españoles se manejan con estas plataformas, aunque represente un desafío desconocido y peligroso para el conjunto del sector financiero.

Es de saber que las Bigtech se centran en brindar servicios como transferencias bancarias y pagos con tarjeta y créditos al consumo; no obstante y dado el alcance obtenido hasta ahora, pronto extenderán la oferta a todo tipo de servicios o productos financieros, tales como: hipotecas, préstamos personales, entre otros, además contemplarán los fondos de inversión, puesto que estos ya están alcance por internet. Por otro lado, Vives (2019) agrega que las Bigtech cuentan con la mayoría de las ventajas de las Fintech, pero sin prácticamente ninguno de sus inconvenientes (con excepción de las dos últimas desventajas ilustradas en el cuadro $\mathrm{N}^{\circ}$ 1. Asimismo, disponen de una base de clientes fieles adecuadamente establecida, muchos datos sobre la reputación elevada y marca potente que serían utilizados desde la red; por otra parte, tienen capacidad de presión y pueden financiar sus actividades con un reducido costo de capital; 
por ello, las Bigtech presentan un potencial de disrupción muy superior para el negocio de la banca tradicional.

Volviendo la mirada a lo anterior, se puede inferir que el impacto de ambos tipos de entidades no bancarias (Fintech y Bigtech) se convierte en organizaciones que ofertan soluciones de pago, así como prestación de servicios, asesoramiento en mercados de capitales, además son un referente en la reconfiguración de las expectativas de los consumidores. En otros segmentos de la banca minorista, especialmente en creación y distribución de créditos al consumo y a las Pymes, el efecto no se visualiza claramente. En síntesis, la tecnología digital genera transformaciones en tramitación general (backoffice), gestión de clientes, análisis de datos, entre otros.

\section{Digitalización de operaciones financieras mediante equipos móviles inteligentes}

Para Yamakawa, Guerrero \& Rees, (2013), las operaciones y servicios financieros ahora pueden realizarse utilizando la denominada banca móvil; la cual está constituida por una serie de aplicaciones tecnológicas que ayudan a superar las limitaciones de espacio y tiempo de las actividades bancarias, no existiendo barreras para la limitar el acceso a las transacciones. Estas aplicaciones tecnológicas, siendo compatibles con el estilo de vida de cada quien y de notable facilidad de uso e innovación personal, contribuyen a aumentar la intención de usanza de estos servicios.

En este mismo tenor, Ciriaco (2003), señala que la digitalización de la banca ha conllevado al uso de los móviles con tecnología GSM para realizar las transacciones financieras. Esto ha sido denominado a nivel mundial como Mobile Banking o banca móvil, que no es más que un banco al alcance y a la mano de cualquier usuario de la tecnología GSM. En concreto, el Mobile Banking provee un puente inmediato entre la institución financiera y el mundo de consumidores móviles, ofreciéndole a los clientes el manejo con seguridad y eficacia de sus asuntos financieros. 
Por su parte, Cedeño-Luna, Alcívar, \& Ponce, (2017) opinan que los dispositivos móviles, tales como: Smartphone (teléfonos inteligentes) y las tablets, son los sistemas promotores de la transformación de las formas de relación de las personas con las organizaciones bancarias; además, estos sistemas están abriendo innumerables oportunidades para diferentes sectores de la sociedad, asimismo, representa una gran tendencia y utilidad para el sector empresarial.

Para cerrar y en virtud de las ideas planteadas se puede decir que la digitalización de las operaciones bancarias, surten el efecto de "banco en la mano"; lo que significa, que se facilitan las transacciones bancarias a través de la telefonía celular, tablets, entre otros dispositivos; causando un efecto transformador, puesto que esta digitalización se constituye en una alternativa que proporciona simplificación de los procesos y actividades en la vida de las personas y de las organizaciones, más aun en los actuales momentos que se vive un proceso de distanciamiento social forzoso producto de la pandemia por Covid-19.

\section{Riesgos de la Digitalización Bancaria}

Resulta pertinente el abordaje de los riesgos a los que se enfrenta el sector bancario ante este proceso de digitalización; al respecto Toloba \& Del Río (2020), comentan que la pervivencia futura de la banca está supeditada en gran medida a la disponibilidad de los sistemas, así como, a su funcionamiento adecuado para ser capaces de cubrir todas las expectativas del negocio, ya sean internas como externas. De ahí que, la digitalización de la banca apareja un incremento del grado de dependencia de su tecnología; esto, aunado al número de activos informáticos que se han de gestionar, su complejidad, aumento de interconexiones entre las mismas entidades y terceros, han agudizado la exposición a ciber amenazas.

A lo anterior se le agrega, que la creciente utilización de datos ya sean estructurados y no estructurados, y de distintas procedencias, pueden aumentar los riesgos debido a diferentes circunstancias como: el uso no autorizado de datos personales de los clientes, 
los comportamientos no éticos de empleados y el asesoramiento sesgado hacia intereses no alineados con los del cliente.

En el mismo orden de ideas, se puede advertir que actualmente el sector financiero experimenta riesgos diferentes al de hace años. En tal sentido, se prevé que la configuración del mapa de riesgos sea distinta ya que la banca se enfrenta a riesgos derivados de la transformación digital, la cual incluye nuevos competidores, ciberseguridad, además de los criptoactivos, aunado a problemas del marco de lucha contra la financiación del crimen organizado, la legitimación de capitales y los nuevos retos de naturaleza medioambiental, social y del gobierno corporativo (Sánchez, 2020). Finalmente, los riesgos y retos a los que se enfrentan los bancos son altamente significativos; sin embargo, en el sector hay conciencia para lograr avances en la gestión de los riesgos y desafíos actuales, lo cual representa la mejor estrategia al alcance para conseguir las oportunidades que surgen de los cambios sociales, económicos y tecnológicos del momento.

\section{REFLEXIONES DE CIERRE}

Una vez realizado el proceso de análisis e interpretación en torno a la transformación bancaria en tiempos de COVID-19 presumiendo un reto organizacional para la gerencia del siglo $\mathrm{XXI}, \mathrm{y}$, en atención al propósito investigativo, se declaran los siguientes enunciados de manera no conclusiva, pues deber seguir siendo objeto de reflexión continua:

La banca experimenta inminentes cambios en la oferta de los servicios financieros, más aún en un contexto como el actual donde los servicios digitales proporcionan ciber seguridad ya que forzosamente impera el distanciamiento social.

En el proceso de digitalización financiera de la banca, la cual ha sufrido una metamorfosis similar en otros países, los instrumentos de pago han jugado un rol destacado; pues, la 


\section{Raysa Chirino-García; Melquiades Salas-Anteliz; Wilfred Vilardy-Naggles}

digitalización no solo representa un complemento para los servicios bancarios, sino que es en sí misma una alternativa con efectos potencialmente eficaces.

Con la intensa transformación de sus actividades, la banca ha tenido que reinventarse tecnológicamente y reajustar sus plataformas electrónicas y así poder atender las exigencias de los usuarios en cuanto a productos y servicios digitales; puesto que, la penetración de Internet aunado a la telefonía móvil han producido cambios en los hábitos y preferencias de los consumidores, quienes interactúan cada vez con mayor frecuencia y de forma continua con el comercio y las transacciones electrónicas.

En el proceso de digitalización, la metamorfosis bancaria apareja cambios asociados a la conversión de una infraestructura modular y flexible que permita integrar el desarrollo de nuevas tecnologías; al mismo tiempo que le de mayor agilidad al crecimiento, creación y mejoramiento de los productos. Esto tiende a beneficiar las inversiones en tecnología y la adopción de estrategias digitales que suponen modificaciones estructurales y organizacionales profundas, con el fin de satisfacer las necesidades de los clientes y/o clientes potenciales.

La banca confronta desafíos y riesgos significativos pues existen situaciones en las que el sector financiero omite controles para las operaciones digitales, constituyéndose esto en una amenaza latente que atenta la ciberseguridad. Por consiguiente, la gerencia debe iniciar procesos de mejoras en los riesgos que incluya la concientización de los clientes internos y externos, en beneficio de ambas partes y a fin de lograr avances en la gestión y ofrecer productos y servicios de calidad.

Dado que las medidas de confinamiento causadas por la pandemia del Covid-19 seguirá por algún tiempo a nivel mundial, es menester que la Gerencia actual de las organizaciones de los distintos ámbitos de la economía y sobre todo las del sector financiero y bancario, asuma retos para poder ofrecer a los clientes servicios con eficiencia, eficacia y calidad haciendo hincapié en el uso herramientas digitales, así como el acceso al mercado electrónico. Aunque, al mismo tiempo, deben empezar a considerar 
las estrategias más adecuadas para ir levantando gradualmente las restricciones, en donde imperará una "nueva normalidad", la cual debe ser enfrentada por la gerencia organizacional con resiliencia asumiendo y enfrentando los desafíos de un retorno a la normalidad que ya no será igual, diseñando estrategias laborales, identificando las oportunidades provenientes de los aprendizajes dejados por la pandemia, cambio de cultura organizacional.

En síntesis, la Gerencia del siglo XXI en el ámbito financiero y bancario deberá cerciorarse de efectuar una evaluación exhaustiva de los retos y éxitos experimentados en tiempos de COVID-19, para no reeditar la vulnerabilidad a la que estuvieron expuestas las instituciones financieras debido a la falta de proactividad y visión prospectiva, a propósito de abastecer la oferta y demanda de productos y servicios con calidad y haciendo hincapié en el uso herramientas digitales, que ofrece el sector a los clientes.

\section{REFERENCIAS CONSUTADAS}

Aldana Zavala, J. J. (2019). La competencia epistemológica en el investigador social universitario venezolano. Praxis, 15(1), 103-115. https://revistas.unimagdalena.edu.co/index.php/praxis/article/view/3091

Antúnez Sánchez, A. (2019). La industria 4.0. Análisis y estudio desde el Derecho en la 4ta Revolución Industrial. Advocatus, 16, 32: 103-131. DOI: https://doi.org/10.18041/0124-0102/a.32.5526.

Arias, F. (2012). El proyecto de investigación: Introducción a la metodología científica. Caracas. Venezuela. Editorial Episteme.

Avendaño, O. (2018). Los Retos de la Banca Digital en México. IUS. Revista del Instituto de Ciencias Jurídicas de Puebla. 12, 41 87-108. https://n9.cl/24l07

Barrutia, J. \& Echebarria, C (2001). Impacto de las nuevas tecnologías en las pautas de consumo de los clientes bancarios y en la propuesta de valor de las entidades. Ekonomiaz: Revista vasca de economía, №. 48, págs. 364-395. 
Universidad Nacional Experimental Francisco de Miranda (UNEFM). Santa Ana de Coro. Venezuela

Raysa Chirino-García; Melquiades Salas-Anteliz; Wilfred Vilardy-Naggles

Boyle, B y Nichols, M. (2020) ¿Cómo COVID-19 acelerará la transformación de los bancos de inversión? Ernst \& Young Global Limited (EYG) publicación del 30/03/2020. https://n9.cl/z7ubi

Bueno Campos, E; Longo Somoza, M; Paz Salmador, M; Morcillo, P. (2017). La Innovación del Modelo de Negocio Bancario: El Reto de la Banca Digital. AECA: Revista de la Asociación Española de Contabilidad y Administración de Empresas, № 120, 3-6. https://n9.cl/uhv71

Capgemini woldwide (2016). Anual Report. https://www.capgemini.com

Carbó, S. (2017). Mitos y realidades de la digitalización financiera: los medios de pago como paradigma. Mediterráneo económico. №. 29, 139-149. https://dialnet.unirioja.es/servlet/articulo?codigo $=6025447$

Casilda, R. (2020). Los Bancos en Evolución. Boletín Económico de Ice (Información Comercial Española). № $3119 . \quad$ 43-56. https://dialnet.unirioja.es/servlet/articulo?codigo=7230199

Cedeño-Luna, M., Alcívar, K. \& Ponce, D. (2017). Observaciones acerca de los dispositivos móviles. Dominio de las Ciencias. Vol. 3, № 4, 89-103. https://dialnet.unirioja.es/servlet/articulo?codigo $=6155633$

Ciriaco, M. (2003). Sistema para realizar transacciones bancarias a través de una red móvil GSM. Télématique, Vol. 2, №. 1, 1-5. https://www.redalyc.org/pdf/784/78420101.pdf

Cuesta, C., Ruesta, M., Tuesta, D. \& Urbiola, P. (2015). La transformación digital de la banca. Observatorio Economía Digital. BBVA Research. https://n9.cl/0y3a

Fanjul Suárez, J.; Valdunciel Bustos, L. (2007). Un paradigma de la nueva economía: el sector financiero en España. Pecunia: Revista de la Facultad de Ciencias $\begin{array}{lllll}\text { Económicas } & y & \text { Empresariales, } & \text { 27-47. }\end{array}$ https://dialnet.unirioja.es/servlet/articulo?codigo $=2392659$

Fontaines, T. (2012). Metodología de la investigación. Pasos para realizar el Proyecto de investigación. Caracas. Venezuela. Júpiter Editores.

Gutiérrez, F. (2016). La Transformación digital de la banca ¿hacia la banca sin bancos? Boletín de estudios económicos, Vol. 71, № 219. 429-456. https://n9.cl/sjjc 
Universidad Nacional Experimental Francisco de Miranda (UNEFM). Santa Ana de Coro. Venezuela

Raysa Chirino-García; Melquiades Salas-Anteliz; Wilfred Vilardy-Naggles

Hempel, M. \& Tur Hartmann, F. (2016). La digitalización de los servicios financieros y su impacto en los medios de pago. Papeles de economía española, № 149, 62-77. https://dialnet.unirioja.es/servlet/articulo?codigo $=5602931$

Igual-Molina, D. (2018). Las fintech y la reinvención de las finanzas. Oikonomics: Revista de economía, empresa y sociedad, №. 10, 2018, págs. 22-44. https://dialnet.unirioja.es/servlet/articulo?codigo $=6972371$

Massanell, J. (2016). La transformación de la banca: reorientación de los canales y servicios digitales. Papeles de economía española. № 19, 1984, 158-165. https://dialnet.unirioja.es/servlet/

Nava, M., Karp, N. \& Nash-Stacey, B. (2014). La paradoja de la generación del milenio. Observatorio bancario Estados Unidos. BBVA Research. https://n9.cl/fpas

Pacheco, L. \& Urbiola, P. (2020). From FinTech to BigTechan evolving regulatory response. BBVA Research. Vol. 75, № 229. 119-141. https://n9.cl/u95v

Palella, S. \& Martins F. (2015). Metodologia de la investigacion cuantitativa. Venezuela. FEDEUPEL

Pejić-Bach, M. (2021). Editorial: Electronic Commerce in the Time of Covid-19 Perspectives and Challenges. Journal of Theoretical and Applied Electronic Commerce Research, 16 (1), $1-1$. https://dialnet.unirioja.es/servlet/articulo?codigo $=7518852$

Reboiro, P (2020). Digitalización del sector bancario. Boletín de estudios económicos, Vol. $75, \quad № \quad 229,53-77$. https://dialnet.unirioja.es/servlet/articulo?codigo $=7430384$

Sánchez, R. (2020). Un Mapa de Riesgos Bancario en transición. Economistas. № Extra 167-168, 66-79. https://dialnet.unirioja.es/servlet/articulo?codigo=7333188

Toloba, C. \& Del Río, J. (2020). La Perspectiva de la Digitalización de la Banca Española: Riesgos y Oportunidades. Revista de Estabilidad Financiera, № 38, 79-97. https://dialnet.unirioja.es/servlet/articulo?codigo $=7430367$ 
Tramazaygues, P (2016). Nuevo ecosistema digital en el sector de los pagos. Papeles de economía española. № $149, \quad 78-92$. https://dialnet.unirioja.es/servlet/articulo?codigo $=5602932$

Vives, X. (2019). La Banca frente a la disrupción digital. Papeles de economía española. № $\quad 162, \quad 2-13$. https://dialnet.unirioja.es/servlet/articulo?codigo $=7244954$

Yamakawa, P, Guerrero, C, Rees, G. (2013). Factores que influyen en la utilización de los servicios de banca móvil en el Perú. Revista Universidad \& Empresa No. 15 (25), 131-149. https://n9.cl/ei6p1 